\title{
Fixed Versus Flexible Antagonist Protocol in Reduced Ovarian Reserve Patients: A Historical Cohort Study
}

\author{
Firouzeh Akbari Asbagh $^{1}{ }^{\mathbb{D}}$, Zahra Dehbashi $^{1^{*}}{ }^{\mathbb{D}}$, Mahmood Moosazadeh $^{2}$, Amirhossien Pourmand $^{3}$, \\ Mahbod Ebrahimi ${ }^{1}$, Naghmeh Pourmand ${ }^{4}$, Parvin Akbari Asbagh ${ }^{5}$
}

\begin{abstract}
Objectives: The antagonist of gonadotropin-releasing hormone is proven to be effective and safe in preventing a surge in luteinizing hormone (LH) during in vitro fertilization (IVF) cycles. In addition, the differentiation of GnRH antagonist protocols is in timing the initiation of the GnRH antagonist. The present study aimed to compare fixed versus flexible antagonist regimens in patients with reduced ovarian reserve in order to identify a better regimen for these patients.

Materials and Methods: This study was of a historical cohort type. Totally, 129 patient files were investigated including 48 versus 66 files related to fixed or flexible protocols, respectively. The mean of age, body mass index (BMI), follicle stimulating hormone (FSH), and anti-Mullerian hormone (AMH), as well as the mean dose of gonadotropin, the mean length of IVF/ICSI cycles, and the mean counts of oocytes and embryos with grading including good, fair, and poor were compared between the 2 groups.

Results: The total and mean consumption of gonadotropin dose (recombinant FSH) in the flexible and fixed groups were significantly different $(P=0.05)$. However, the mean antagonist (cetrotide) doses in the fixed and flexible groups were not significantly different while it was lower in the flexible group $(P=0.50)$. Finally, the duration of gonadotropin consumption in IVF/ICSI period was not significantly different between the two groups.

Conclusions: In general, the fixed protocol is recommended in patients with reduced ovarian reserve since it is simple to use and more cost effective compared to the flexible protocol.

Keywords: Fixed, Flexible, Ovarian
\end{abstract}

\section{Introduction}

The antagonist of gonadotropin releasing hormone $(\mathrm{GnRH})$ is effective and safe for preventing a luteinizing hormone (LH) surge during in vitro fertilization (IVF) cycles $(1,2)$. During the past years, there has been a progressive shift away from GnRH agonist downregulation toward the GnRH antagonist. In IVF/ICSI cycles, the GnRH antagonist competitively binds the $\mathrm{GnRH}$ receptor and yields a rapid onset and withdrawal of functions (3-6). In addition, the differentiation of $\mathrm{GnRH}$ antagonist protocols is believed to be in timing the initiation of the GnRH antagonist $(6,7)$. In a fixed protocol, the GnRH antagonist is initiated in the sixth days of ovarian stimulation versus the flexible protocol follicular diameter as reference points for GnRh antagonist initiation $(6,8,9)$.

Breakthrough LH surge during GnRH-antagonist is not frequently encountered in IVF cycles. These patients have a poorer ovarian reserve, increased age, and poor response to gonadotropins (3). In the Bologna criteria published by European Society of Human Reproduction and Embryology (ESHRE) in 2011, an abnormal ovarian reserve test is identified as antral follicle count (AFC) of less than 5-7 follicles or anti-Mullerian hormone (AMH)

\section{$<0.5-1.1 \mathrm{ng} / \mathrm{mL}(10-13)$}

Tannus et al studied the effect of delayed initiation of gonadotropin-releasing hormone antagonist in a flexible protocol on IVF outcome. This study included patients aged $\leq 40$ years. The antagonist was started when the leading follicle size was $\geq 13 \mathrm{~mm}$ or the serum $\mathrm{E}_{2}$ level achieved $\geq 300 \mathrm{pg} / \mathrm{mL}$ (10).

\section{Objectives \\ Few studies considered fixed versus flexible antagonist regimens in reduced ovarian reserved patients. Further, using the antagonist in reduced ovarian reserve patients is progressive. Therefore, the authors decided to design a study and compare the fixed versus flexible antagonist regimens in reduced ovarian reserve patients in order to identify a better regimen in these patients.}

\section{Design, Participants, and Data Collection Method}

This study reviewed the IVF/ICSI files of the patients investigated in university-based tertiary hospitals in Tehran, Iran. Patients $<41$ years with reduced ovarian reserve, according to Bologna criteria, and those who received an antagonist protocol for IVF/ICSI cycles were included in this research conducted during (October)

Received 1 June 2018, Accepted 2 August 2018, Available online 10 September 2018

${ }^{1}$ Department of Obstetrics and Gynecology, Tehran University of Medical Sciences, Tehran, Iran. ${ }^{2}$ Health Sciences Research Center, Addiction Institute, Mazandaran University of Medical Sciences, Sari, Iran. ${ }^{3}$ School of Medicine, Tehran University of Medical Sciences, Tehran, Iran. ${ }^{4}$ Tehran University of Medical Sciences, Tehran, Iran. ${ }^{5}$ Department of Pediatrics, Tehran University of Medical Sciences, Tehran, Iran.

*Corresponding Author: Zahra Dehbashi, Tel: +982186089076, Email: zddoctor1390@gmail.com 
2013-(September) 2017.

Incomplete patient files were excluded from the study. After the detailed study of the patients' files, the patients were again contacted and necessary information was obtained. Patients with reduced ovarian reserve in our center normally underwent treatment with an antagonist protocol and antagonist (cetrotide) $0.25 \mathrm{mg} / \mathrm{d}$ was initiated in 2 protocols. In one protocol, cetrotide was taken in a fixed protocol in sixth days after starting the gonadotropin. In a flexible protocol, cetrotide initiated to growth in ovarian follicle size. If the largest achieved follicle size was $13 \mathrm{~mm}$, cetrotide was initiated. Each protocol for every patient was selected by a related doctor. A number of 129 patient files were investigated out of which 15 files were excluded as a result of incomplete information. Totally, less than half of the files $(n=48)$ were related to a fixed protocol while the remaining files $(n=66)$ were associated with a flexible protocol. A detailed history of all the patients was recorded. Then, the patients underwent a physical examination. Hormonal study (i.e., follicle stimulating hormone [FSH], $\mathrm{LH}, \mathrm{AMH}$, and $\mathrm{E}_{2}$ ) in 2 or 3 days of menses was recorded. The hysterosalpingographic report, as well as the laparoscopic hysteroscopic surgical report of the patients was available. All the patients in 2 or three days of menstruation underwent transvaginal sonography. Furthermore, gonadotropin drugs (recombinant FSH [Gonal-F, Merk], HMG [Merional or Menogan, Ferring, IBSA], and urinary FSH [Fostimone, IBSA]) were initiated based on the age, AFC, and AMH. The patients had serially transvaginal sonography and the dose of gonadotropins was adjusted. In the fixed group, antagonist (cetrotide, Merk Serono) $0.25 \mathrm{mg} / \mathrm{d}$ was initiated in sixth days after starting the gonadotropin while in the flexible group, if the largest achieved follicle size was $13 \mathrm{~mm}$, then, the cetrotide $0.25 \mathrm{mg} / \mathrm{d}$ was initiated. When the follicle size achieved $18 \mathrm{~mm}$ HCG 10000 IU (Darou Paksh or Organon) or recombinant HCG (Ovitrel) $250 \mu \mathrm{G}$ (Merk Serono) was administered. After 36 hours, oocytes were retrieved. If in 11-13 days of menstruation the follicles failed to grow or the growth was stopped and the $\mathrm{E}_{2}$ level reached $<200 \mathrm{pg} / \mathrm{mL}$, then, the cycle was canceled.

Two to three days after the oocytes were retrieved, the embryos were transferred. After fourteen days, B-HCG was checked. If the gestational sac was observed in the transvaginal sonography, clinical pregnancy was considered positive; however, if B-HCG was positive and the gestational sac was not represented by transvaginal sonography, then, chemical pregnancy was considered.

All patients received luteal phase support by cyclogest $400 \mathrm{mg}$ vaginally twice daily at least 14 days until a pregnancy test was performed. If the pregnancy test was positive, cyclogest $400 \mathrm{mg}$ was administered vaginally twice daily and continued until 10-12 weeks of pregnancy.

\section{Data Analysis}

The mean age, body mass index (BMI), FSH, and AMH were calculated and the groups were compared. The mean dose of gonadotropin and the mean length of IVF/ICSI cycles were compared between the 2 groups. Moreover, the mean count of oocytes was retrieved and the mean count of embryos with grading including good, fair, and poor was compared between the 2 groups.

In this research, statistical analysis was conducted using the SPSS software, version 19. The continuous and categorical variables were employed in the present study. Descriptive statistics were reported as mean and SD for continuous variables and percentage for categorical variables. Additionally, a two-sample $t$ test was used for continuous variables and $\chi^{2}$ analysis or Chi-square test of independence was implemented for categorical variables for between-group comparisons. The odds ratio was estimated with $95 \% \mathrm{CI}$ and the level of significance was considered at $P<0.05$.

\section{Results}

The findings demonstrated no significant difference between the mean age of the patients between the fixed (35.8 years) and the flexible (38.2 years) groups. All patients were $<41$ years old. In addition, the mean BMI in the fixed and flexible groups were 25.19 and 25.95, respectively, and based on the result, no significant difference was observed between both groups in this respect.

The AMH of all the patients was $<0.5-1.1 \mathrm{ng} / \mathrm{mL}$ and there was no significant difference between the 2 groups. Further, the mean of FSH in the fixed group was 7.45 while it was equal to 9.62 in the flexible group and no significant difference was found between the 2 groups in this regard (Table 1) (Figure 1). Other causes of infertility (i.e., mal, tubal, and endometriosis) were studied between the 2 groups; however, there was no statistically significant difference between both groups (Table 2).

The count of oocytes $(P=0.38)$ and embryos $(P=0.51)$, the number of embryos with different grades including the good $(P=0.12)$, fair $(P=0.18)$, and poor $(P=0.19)$ were not significantly different between the fixed and flexible groups (Figure 1) ( Table 3).

Furthermore, as shown in Table 3, endometrial thickness is not significantly different between the 2 groups $(P=0.26)$.

The total and mean consumption of gonadotropin dose (recombinant FSH) in the flexible group was

Table 1. The Comparison of Clinical Characteristics Between the Fixed and Flexible Groups

\begin{tabular}{lccccc}
\hline \multirow{2}{*}{ Variables } & \multicolumn{2}{c}{ Fix Group } & \multicolumn{2}{c}{ Flexible Group } & \multirow{2}{*}{$\boldsymbol{P}$ Value } \\
\cline { 2 - 5 } & Mean & SD & Mean & SD & \\
\hline Age & 35.87 & 3.76 & 38.42 & 5.07 & 0.24 \\
BMI & 25.19 & 1.93 & 25.95 & 5.29 & 0.35 \\
FSH & 7.45 & 3.97 & 9.62 & 5.36 & 0.38 \\
AMH & 0.70 & 0.33 & 0.52 & 0.36 & 0.29 \\
\hline
\end{tabular}

Note. SD: standard deviation; BMI: body mass index; FSH: follicle stimulating hormone; $\mathrm{AMH}$ : anti-Mullerian hormone. 
Table 2. The comparison of the Causes of Infertility Between the 2 Groups

\begin{tabular}{lccc}
\hline Variables & Fixed & Flexible & $\boldsymbol{P}$ Value \\
\hline Mal & $25 \%$ & $41 \%$ & 0.44 \\
Tubal & $12 \%$ & $17 \%$ & 0.80 \\
Endometriosis & $12 \%$ & $8 \%$ & 0.76 \\
\hline
\end{tabular}

Table 3. The Comparison of Oocyte Count and the Quality of Fetus Between the Fixed and Flexible Groups

\begin{tabular}{|c|c|c|c|c|c|}
\hline \multirow{2}{*}{ Variables } & \multicolumn{2}{|c|}{ Fixed Group } & \multicolumn{2}{|c|}{ Flexible Group } & \multirow{2}{*}{$P$ Value } \\
\hline & Mean & SD & Mean & SD & \\
\hline Oocyte number & 3.00 & 2.07 & 2.08 & 2.35 & 0.38 \\
\hline Embryo number & 1.75 & 1.28 & 1.27 & 1.68 & 0.51 \\
\hline Good embryo & 2.33 & 0.82 & 1.33 & 1.21 & 0.12 \\
\hline Fair embryo & 1.98 & 0.96 & 1.00 & 1.41 & 0.19 \\
\hline Poor embryo & 2.18 & 1.80 & 1.25 & 2.50 & 0.18 \\
\hline blastocyst & 0.00 & 0.00 & 1.25 & 2.50 & 0.44 \\
\hline $\begin{array}{l}\text { Endometrial } \\
\text { thickness }\end{array}$ & 7.71 & 1.70 & 5.71 & 4.19 & 0.26 \\
\hline
\end{tabular}

higher compared to the fixed group and this finding was significantly different between the 2 groups $(P=0.05)$. Although the mean of antagonist (cetrotide) doses in the fixed group was more than that of the flexible group, it was not significantly different $(P=0.50)$.

Moreover, Table 4 demonstrates that the duration of gonadotropin consumption in IVF/ICSI between both groups is not significantly different $(P=0.3)$. The comparison of gonadotropin consumption and length of treatment between the 2 groups is illustrated in Figure 2 .

Based on the results represented in Table 5, the chemical and clinical pregnancy rate between the 2 groups are not significantly different $(P=0.23)$. However, the rate of the cycle canceled in the flexible group is higher compared to the fixed group; however, it is not significantly different $(P=0.07)$.

\section{Discussion}

The present study compared fixed and flexible antagonist protocols on reduced ovarian reserve patients. The age

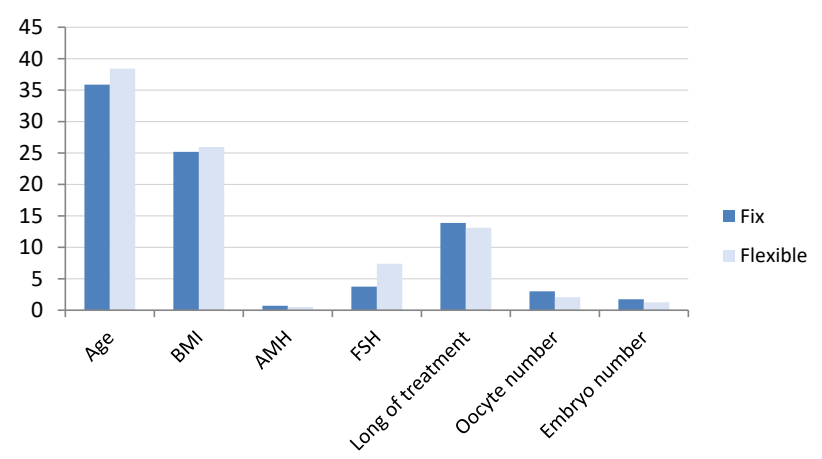

Figure 1. The Comparison of the Clinical Characteristics and Some IVF/ ICSI Outcomes Between the Fixed and Flexible Groups.
Table 4. The Comparison of Gonadotropin and Antagonist (Cetrotide) Consumption Between the Fixed and Flexible Groups

\begin{tabular}{lccccc}
\hline \multirow{2}{*}{ Variables } & \multicolumn{2}{c}{ Fixed } & \multicolumn{2}{c}{ Flexible } & $\boldsymbol{P}$ \\
\cline { 2 - 5 } & Mean & SD & Mean & SD & Value \\
\hline Long of treatment & 13.87 & 0.99 & 13.12 & 1.73 & 0.30 \\
HMG & 4.37 & 2.67 & 5.25 & 8.51 & 0.78 \\
FSH recombinant & 15.50 & 15.42 & 34.83 & 18.04 & 0.02 \\
Urinary FSH & 10.12 & 19.17 & 5.67 & 19.63 & 0.62 \\
SUM1=HMG+FSH & 30.00 & 15.16 & 45.75 & 17.31 & 0.05 \\
recom+ urinary FSH & 4.22 & 1.09 & 3.44 & 3.17 & 0.50 \\
Cetrotide & & & & &
\end{tabular}

Note. SD: Standard deviation; HMG: Human menopausal gonadotropin; FSH: Follicle stimulating hormone.

and BMI of the patients in both groups represented no significant difference. Additionally, the FSH and AMH of the patients demonstrated no significant difference. In addition, the counts of oocytes $(P=0.38)$, embryo $(P=0.51)$, and embryo with good $(P=0.12)$, fair $(P=0.18)$, and poor $(P=0.19)$ grades, along with endometrial thickness were not significantly different between both groups. However, the mean consumption of gonadotropin dose in the flexible group was more than that of the fixed group $(P=0.05)$, indicating that there was a significant difference between the 2 groups in this respect.

Conversely, the length of the gonadotropin consumption was not significantly different in the fixed and flexible groups. Although the mean antagonist (cetrotide) doses in the fixed group were more than those of the flexible group, they were not significantly different $(P=0.50)$. The chemical and clinical pregnancy rate indicated no significant difference between the fixed and flexible groups. However, the rate of the cycle cancelled in the flexible group was higher compared to the fixed group, however, it was not significantly different $(P=0.07)$.

Tannus et al considered the effect of delayed starting of gonadotropin-releasing hormone antagonist in a flexible protocol on the IVF outcome. In this study, only the patients $\leq 40$ years were included. The administration of the antagonist was started when the leading follicle size

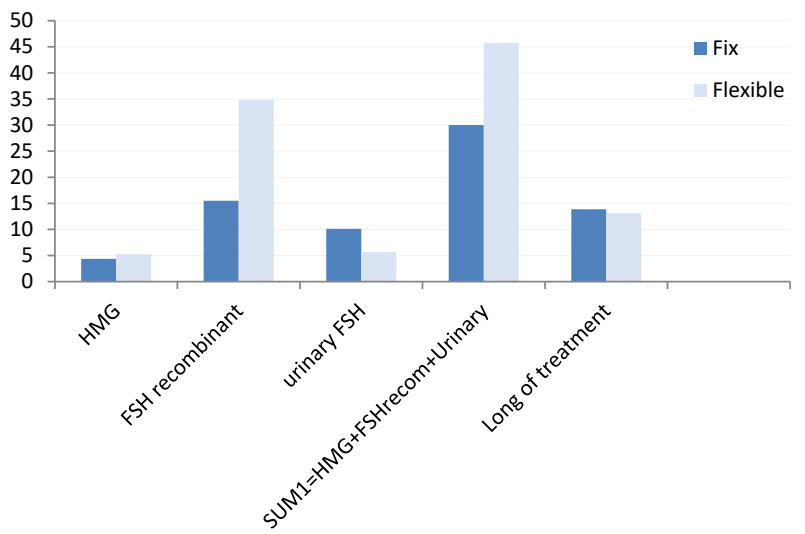

Figure 2. The Comparison of Gonadotropin Consumption and the Length of Treatment Between the Fixed and Flexible Groups 
Table 5. The Comparison of Pregnancy Outcome and Cycle Canceled Between the Fixed and Flexible Groups

\begin{tabular}{lccc}
\hline Variables & Fix Group & Flexible Group & $\boldsymbol{P}$ Value \\
\hline Clinical pregnancy & $10 \%$ & $8 \%$ & 0.23 \\
Chemical pregnancy & $12 \%$ & $10 \%$ & 0.23 \\
Cycle cancel & $22 \%$ & $33 \%$ & 0.07 \\
\hline
\end{tabular}

was $\geq 13 \mathrm{~mm}$ or the serum $\mathrm{E}_{2}$ level achieved $\geq 300 \mathrm{pg} / \mathrm{mL}$ (10). The patients were divided into 2 groups based on the day of starting the antagonist administration. As regards the time of starting GnRH antagonist administration, the results represented no difference in implantation, as well as clinical and ongoing pregnancy rate (10). These findings are in line with the results of the present study; however, the current study only investigated the reduced ovarian reserve patients while patients $<41$ years were included. Further, in the above-mentioned study, a higher dose of gonadotropin was administered in the group receiving the antagonist, initiated later than sixth days after starting gonadotropin, and the time of gonadotropin administration was longer.

In the present research, the dose of gonadotropin was higher in the flexible group while the time of gonadotropin administration was not different between both groups although there was a difference in the definition of the flexible regimen and the method of separating both groups.

Estratios compared fixed and flexible antagonist protocols in IVF/ICSI cycles. In this study, i8t was included patients with age $<39$ years while those patients who responded poorly were excluded from the study. The findings revealed that flexible antagonist administration failed to reduce the incidence of $\mathrm{LH}$ surge compared to the fixed antagonist protocol and that in the fixed group, antagonist consumption was lower. Then, cetrorelix was started on sixth days of rFSH stimulation in the fixed group whereas in the flexible group, cetrorelix was started when at least one of the following criteria were found: $\mathrm{LH}$ $>10$ IU/L, presence of a follicle with mean diameter $>12$ $\mathrm{mm}$, and serum $\mathrm{E}_{2}$ level $>150 \mathrm{pg} / \mathrm{mL}$ (14). Clinical and ongoing pregnancy rates were not significantly different between the 2 groups. This finding is in conformity with that of the present study; however, the included flexible criteria were different. Based on the results of the current study, the dose of gonadotropin consumption in the fixed group was lower. However, in this study, only reduced ovarian reserve patients were included while Estratios $M$ (14) excluded this group of patients from the study.

\section{Conclusions}

The findings of the present study demonstrated that the flexible regimen in reduced ovarian reserve patients was not superior compared to the fixed regimen in terms of endometrial thickness and clinical pregnancy rate; however, the dose of gonadotropin administration in the flexible group was higher. Furthermore, the mean antagonist (cetrotide) doses in the fixed group was higher than that of the flexible group; however, these findings were not significantly different. In general, the fixed protocol is recommended in reduced ovarian reserve patients since it is cost effective compared to the flexible protocol and simple to use because it requires fewer transvaginal sonography for determining the size of the follicle.

\section{Conflict of Interests}

Authors declare that they have no conflict of interests.

\section{Ethical Issues}

This study, as a historical cohort, was confirmed by the Ethical Committee of Tehran University of Medical Sciences.

\section{Financial Support}

None.

\section{Acknowledgments}

The authors would like to thank the patients, as well as the Infertility Department of Tehran University of Medical Sciences for their help.

\section{References}

1. Diedrich K, Diedrich C, Santos E, et al. Suppression of the endogenous luteinizing hormone surge by the gonadotrophin-releasing hormone antagonist Cetrorelix during ovarian stimulation. Hum Reprod. 1994;9(5):788791.

2. Sbracia M, Colabianchi J, Giallonardo A, et al. Cetrorelix protocol versus gonadotropin-releasing hormone analog suppression long protocol for superovulation in intracytoplasmic sperm injection patients older than 40. Fertil Steril. 2009;91(5):1842-1847. doi:10.1016/j. fertnstert.2008.02.165

3. Reichman DE, Zakarin L, Chao K, Meyer L, Davis OK, Rosenwaks Z. Diminished ovarian reserve is the predominant risk factor for gonadotropin-releasing hormone antagonist failure resulting in breakthrough luteinizing hormone surges in in vitro fertilization cycles. Fertil Steril. 2014;102(1):99-102. doi:10.1016/j. fertnstert.2014.04.010

4. Borm G, Mannaerts B. Treatment with the gonadotrophinreleasing hormone antagonist ganirelix in women undergoing ovarian stimulation with recombinant follicle stimulating hormone is effective, safe and convenient: results of a controlled, randomized, multicentre trial. The European Orgalutran Study Group. Hum Reprod. 2000;15(7):1490-1498.

5. Frydman R, Cornel C, de Ziegler D, Taieb J, Spitz IM, Bouchard P. Prevention of premature luteinizing hormone and progesterone rise with a gonadotropin-releasing hormone antagonist, Nal-Glu, in controlled ovarian hyperstimulation. Fertil Steril. 1991;56(5):923-927.

6. Dayal MB, Frankfurter D, O’Hern C, Peak D, Dubey A, Gindoff PR. The use of lead follicle diameter to initiate gonadotropin-releasing hormone antagonist does not affect 
in vitro fertilization clinical pregnancy, implantation, or live birth rates: a prospective, randomized study. Fertil Steril. 2009;92(6):2047-2049. doi:10.1016/j.fertnstert.2009.05.083

7. Mochtar $\mathrm{MH}$. The effect of an individualized GnRH antagonist protocol on folliculogenesis in IVF/ICSI. Hum Reprod. 2004;19(8):1713-1718. doi:10.1093/humrep/ deh334

8. Ludwig M, Katalinic A, Banz C, et al. Tailoring the GnRH antagonist cetrorelix acetate to individual patients' needs in ovarian stimulation for IVF: results of a prospective, randomized study. Hum Reprod. 2002;17(11):2842-2845.

9. Escudero E, Bosch E, Crespo J, Simon C, Remohi J, Pellicer A. Comparison of two different starting multiple dose gonadotropin-releasing hormone antagonist protocols in a selected group of in vitro fertilization-embryo transfer patients. Fertil Steril. 2004;81(3):562-566. doi:10.1016/j. fertnstert.2003.07.027

10. Tannus S, Weissman A, Boaz M, et al. The effect of delayed initiation of gonadotropin-releasing hormone antagonist in a flexible protocol on in vitro fertilization outcome. Fertil Steril. 2013;99(3):725-730. doi:10.1016/j. fertnstert.2012.11.020

11. Younis JS, Ben-Ami M, Ben-Shlomo I. The Bologna criteria for poor ovarian response: a contemporary critical appraisal. J Ovarian Res. 2015;8:76. doi:10.1186/s13048015-0204-9

12. Frydman R. Poor responders: still a problem. Fertil Steril. 2011;96(5):1057. doi:10.1016/j.fertnstert.2011.09.051

13. Ferraretti AP, Gianaroli L. The Bologna criteria for the definition of poor ovarian responders: is there a need for revision? Hum Reprod. 2014;29(9):1842-1845. doi:10.1093/ humrep/deu139

14. Kolibianakis EM, Venetis CA, Kalogeropoulou L, Papanikolaou E, Tarlatzis BC. Fixed versus flexible gonadotropin-releasing hormone antagonist administration in in vitro fertilization: a randomized controlled trial. Fertil Steril. 2011;95(2):558-562. doi:10.1016/j. fertnstert.2010.05.052

(C) 2019 The Author (s); This is an open-access article distributed under the terms of the Creative Commons Attribution License (http://creativecommons.org/licenses/by/4.0), which permits unrestricted use, distribution, and reproduction in any medium, provided the original work is properly cited. 